\title{
REPETIBILIDADE DE CARACTERES DO CACHO DE AÇAIZEIRO NAS CONDIÇÕES DE BELÉM-PA ${ }^{1}$
}

\author{
MARIA DO SOCORRO PADILHA DE OLIVEIRA² E GUILHERME LEOPOLDO DA COSTA FERNANDES ${ }^{2}$
}

\begin{abstract}
RESUMO - Estimou-se o coeficiente de repetibilidade em caracteres do cacho de açaizeiro (Euterpe oleracea Mart.) nas condições de Belém-PA, com o intuito de determinar a capacidade de eles expressarem a variabilidade genética dessa fruteira. Para tanto, foram colhidos quatro cachos por planta, todos apresentando completa maturação, em 30 genótipos pertencentes à Coleção de Germoplasma de Açaí da Embrapa Amazônia Oriental, onde foram mensurados seis caracteres: peso total do cacho (PTC), peso de frutos por cacho ( $\mathrm{PFC}$ ), número de frutos por cacho (NFC), número de ráquilas por cacho (NRC), peso médio do fruto (PMF) e rendimento de frutos por cacho (RFC). A análise da repetibilidade, do número de medições necessárias e do coeficiente de determinação para cada caráter foi obtida através do método da análise de variância, utilizando o modelo com dois fatores de variação. Verificou-se que todos os caracteres apresentaram diferenças significativas entre genótipos, com o número de frutos, número de ráquilas e peso médio do fruto, evidenciando diferenças ao nível de $1 \%$ de probabilidade. Porém, o maior coeficiente de repetibilidade foi registrado para peso médio do fruto, enquanto os demais caracteres apresentaram valores inexpressivos. Essa variável teve, também, o maior coeficiente de determinação; entretanto, o número de repetições desejável para esse caráter deve ser quase o triplo do usado nesse estudo. Pelo fato de o coeficiente de repetibilidade expressar o valor máximo de herdabilidade, conclui-se que o PMF pode ser usado como parâmetro de seleção em métodos de melhoramento menos rigorosos.
\end{abstract}

Termos para indexação: variância genética, variância ambiental, herdabilidade, seleção.

\section{REPEATIBILITY OF CHARACTERS OF THE RACEME OF AÇAI PALM AT BELÉM-PA}

\begin{abstract}
Was considered the repeatibility coefficient in characters of raceme in açai palm (Euterpe oleracea Mart.) at Belém-PA with the intention of determining their capacity to express the genetic variability of that fruit bowl. For that were picked four racemes by plant, all presenting complete maturation, in 30 genotypes belonging to the Collection of Germplasm of Açai palm of Oriental Amazonian Embrapa, where six characters were evaluated: weight of the raceme (PTC), weight of fruits per raceme (PFC), number of fruits per raceme (NFC), rachilles number per raceme (NRC), weigh medium of the fruit (PMF) and revenue of fruits ber raceme (RFC). The analysis of the repeatibility, of the number of necessary measurements and of the coefficient determination for each character was obtained through the method of the variance analysis using the model with two variation factors. It was verified that all the characters presented significant differences among genotypes, with the number of fruits, rachilles number and medium weight of the fruit being at the level of $1 \%$ of probability. However, the largest repeatibility coefficient was registered for medium weight of the fruit, while the others presented inexpressive values. That variable also had the largest determination coefficient. Meanwhile to the number of replications desirable for that character should be almost the triple of used in this study. The fact of the repeatibility coefficient expresses the maximum value of heritability can be concluded that PMF could be used as a selection parameter in less rigorous improvement methods.
\end{abstract}

Index terms: genetic variance, environmental variance, heritability, selection.

\section{INTRODUÇÃO}

O açaizeiro (Euterpe oleracea Mart.) é uma fruteira nativa da Amazônia que vem conquistando o mercado nacional mediante comercialização da bebida "in natura" e congelada obtida pelo processamento da parte comestível de seus frutos, denominada de "açaí".

Com o aumento do mercado de açaí, muitos produtores têm procurado órgãos de pesquisa para obter informações sobre seu cultivo e, principalmente, sobre sementes de qualidade comprovada. Em vista da necessidade de fornecer material básico para plantio, a seleção de plantas matrizes, através de características fenotípicas, tem sido uma das alternativas possíveis. Um dos pontos cruciais nesse processo tem sido a obtenção de parâmetros que apresentem herdabilidade alta para que se possam obter consideráveis ganhos genéticos.

No açaizeiro, os frutos encontram-se inseridos em cachos ou infrutescências, constituindo-se como a principal parte da planta para o mercado. Vale ressaltar que, na maioria das fruteiras perenes, os experimentos ocupam grandes áreas, sendo comum instalação sem delineamento experimental, tornando-se difícil a obtenção da herdabilidade, como é o caso do açaizeiro. Assim, o coeficiente de repetibilidade surge como uma alternativa nesses experimentos, por permitir tomar mais de uma medida no 
mesmo indivíduo, definindo o limite superior da herdabilidade e do grau de determinação (Vencovsky, 1973; Falconer, 1975; Cruz \& Regazzi, 1997).

O coeficiente de repetibilidade tem sido empregado em variáveis de produção das seguintes fruteiras perenes: coqueiro (Siqueira, 1982), cupuaçuzeiro (Costa et al.,1997) e aceroleira (Lopes et al., 2000).

Este trabalho teve como objetivo estimar, para as condições de Belém-PA, o coeficiente de repetibilidade em caracteres do cacho de açaizeiro, assim como o número de medições ideal e o coeficiente de determinação.

\section{MATERIAL E MÉTODOS}

Neste estudo, foram utilizados 30 genótipos de açaizeiro selecionados ao acaso, na Coleção de Germoplasma da Embrapa Amazônia Oriental, Belém-PA, os quais foram instalados nessa coleção em novembro de 1985, no espaçamento de $5 \mathrm{~m}$ x 3m, em condições de terra firme, em solo do tipo Latossolo Amarelo textura média.

De cada planta, foram colhidos quatro cachos consecutivos após atingirem a completa maturação, os quais foram identificados e levados ao Laboratório de Fitomelhoramento da Embrapa Amazônia Oriental, para a mensuração de seis caracteres: peso total do cacho (PTC), peso de frutos por cacho ( $\mathrm{PFC}$ ), expressos em $\mathrm{kg}$, número de ráquilas por cacho (NRC), número de frutos por cacho (NFC), peso médio do fruto (PMF) expresso em g, e rendimento de frutos por cacho (RFC) em percentagem.

As análises estatísticas, para todos os caracteres, foram efetuadas pelo método da análise de variância com dois fatores de variação, seguindo a metodologia de Cruz \& Regazzi (1997). Os dados obtidos em unidades, como número de ráquilas por cacho (NRC) e número de frutos por cacho (NFC), foram transformados em $\sqrt{\mathrm{x}}$, enquanto os de percentagens foram transformados em $\sqrt{\mathrm{x} / 100}$.

O modelo estatístico adotado foi:

$\mathrm{y}_{\mathrm{ij}}=\mathrm{m}+\mathrm{g}_{\mathrm{i}}+\mathrm{c}_{\mathrm{j}}+\mathrm{e}_{\mathrm{ij}}$, onde:

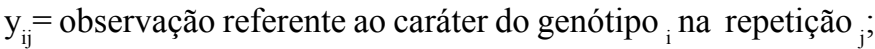
$\mu=$ média geral;

$\mathrm{g}_{\mathrm{i}}=$ efeito aleatório do genótipo i sob a influência do ambiente permanente $\left({ }_{\mathrm{i}=1,2, \ldots, 30}\right)$;

$c_{j}=$ efeito fixo da medição realizada no cacho ${ }_{j}\left({ }_{j=1,2, \ldots, 4}\right) ;$

$\mathrm{e}_{\mathrm{ij}}=$ erro experimental.

As estimativas dos componentes de variância foram obtidas por meio das esperanças dos quadrados médios presentes na Tabela 1, e o coeficiente de repetibilidade estimado pela fórmula :

$$
\mathrm{r}={\sigma_{\mathrm{g}}^{2}}_{\mathrm{g}_{\mathrm{e}}}{\sigma_{\mathrm{e}}^{2}}+\sigma_{\mathrm{g}}^{2}, \text { onde: }
$$

$\mathrm{r}=$ coeficiente de repetibilidade;

$\sigma^{2}=$ estimativa da variância entre genótipos;

$\sigma_{\mathrm{e}}{ }^{\mathrm{g}}=$ estimativa da variância do erro experimental.
O cálculo do número de medições necessárias $\left(\eta_{\mathrm{o}}\right)$ para predizer o valor real dos indivíduos a $95 \%$ de determinação foi obtido pela fórmula:

$\eta_{\mathrm{o}}=\mathrm{R}^{2} \cdot(1-\mathrm{r}) /\left(1-\mathrm{R}^{2}\right) \cdot \mathrm{r}$, onde:

$\eta_{\mathrm{o}}=$ número de cachos necessários para a determinação genotípica desejável;

$\mathrm{R}^{2}=$ coeficiente de determinação a $95 \%$;

$\mathrm{r}=$ coeficiente de repetibilidade.

O coeficiente de determinação para o número de medições realizadas foi calculado através da fórmula:

$$
\mathrm{R}^{2}=\mathrm{n} . \mathrm{r} / 1+\mathrm{r} .(\mathrm{n}-1) \text {, onde: }
$$

$\mathrm{R}^{2}=$ coeficiente de determinação para o número de repetições testadas;

$\mathrm{n}=$ número de medições efetuadas;

$\mathrm{r}=$ coeficiente de repetibilidade.

\section{RESULTADOS E DISCUSSÃO}

Os quadrados médios para as seis variáveis analisadas encontram-se na Tabela 2. Como se pode verificar, o número de frutos por cacho (NFC), o número de ráquilas por cacho (NRC) e o peso médio do fruto (PMF) apresentaram diferenças significativas ao nível de $1 \%$ de probabilidade entre os genótipos avaliados, enquanto o peso total do cacho (PTC), o peso de frutos por cacho (PFC) e o rendimento de frutos (RFC) foram distintos ao nível de 5\%. Estes valores fornecem indícios da ocorrência de variabilidade genética entre os genótipos para todos as caracteres mensurados.

De um modo geral, os cachos exibiram peso médio desejável $(2,44 \mathrm{~kg})$, possuindo também um considerável rendimento de frutos (73,9\%). Oliveira et al. (1998), estudando acessos de açaizeiro dessa coleção, também obtiveram rendimentos altos (acima de 60\%), levando a crer que, nas condições do estudo, este caráter apresente estabilidade fenotípica.

As estimativas de variância entre genótipos $\left(\sigma_{\mathrm{g}}^{2}\right)$, variância residual $\left(\sigma_{\mathrm{e}}^{2}\right)$, coeficiente de repetibilidade $(\mathrm{r})$, número de medições desejável para predizer o valor real dos indivíduos com $95 \%$ de determinação $\left(\eta_{\mathrm{o}}\right)$ e coeficiente de determinação para as medições efetuadas $\left(\mathrm{R}^{2}\right)$ nos seis caracteres estudados estão contidas na Tabela 3 .

Para os valores da variância entre genótipos, foi constatado que apenas o caráter peso médio do fruto (PMF) exibiu valor superior ao da variância residual. Para os demais caracteres, as variâncias residuais alcançaram valores maiores que as entre genótipos, expressando que tais variáveis se apresentam altamente influenciadas pelas condições ambientais e, portanto, não oferecem subsídios para serem utilizadas em programas de melhoramento.

No caso dos coeficientes de repetibilidade, foi observada uma variação de 0,0303 a 0,6328 . Os caracteres número de ráquilas por cacho (NRC), número de frutos por cacho (NFC), peso total do cacho (PTC), peso de frutos por cacho (PFC) e 
rendimento de frutos (RFC) exibiram valores de 0,$2905 ; 0,2152$; 0,$1550 ; 0,1382$ e 0,0303 , respectivamente, e, como foram considerados baixíssimos e inexpressivos, fornecem indícios da não-existência de regularidade na repetição dessas variáveis de um cacho para o outro.

Por outro lado, o peso médio do fruto (PMF) foi a única variável que alcançou valor considerável $(0,6328)$, indicando regularidade entre as medições, podendo-se então esperar que esta variável consiga expressar um bom controle genético. Para Vencovsky (1973), coeficientes de repetibilidades altos podem ser empregados como parâmetros para medir a capacidade de repetição da expressão do caráter avaliado. Com base nessa constatação, pode-se inferir que o peso médio do fruto pode ser empregado como parâmetro desejável em métodos simples de melhoramento do açaizeiro para frutos, a exemplo da seleção fenotípica simples, com perspectivas de se obter bom ganho genético.

Pelo fato de os principais caracteres relacionados com o mercado de frutos (PFC, NFC e RFC) terem apresentado baixíssimos coeficientes de repetibilidade, indicando que devam ser bastante influenciados pelo ambiente, sugere-se que, no emprego dessas variáveis, sejam propostos métodos de melhoramento com controle parental.
Os coeficientes de determinação para os seis caracteres avaliados, nas condições do estudo, demonstraram confiabilidade apenas para o peso médio do fruto (PMF), que exibiu valor superior a $80 \%$. Os demais caracteres não oferecem respaldo, havendo necessidade de serem realizadas novas avaliações com um número de medições bem maior que as efetuadas. Em vista desses resultados, o número de medições desejáveis para uma seleção mais efetiva no emprego do peso médio do fruto deve ser de, no mínimo, onze cachos, ou seja, quase três vezes a mais que o usado neste trabalho, mas possível de ser realizada sem muito dispêndio. Contudo, para os caracteres mencionados como os mais importantes na produção de frutos, os valores variaram de 69 a 138 medições, dificultando consideravelmente suas aplicações na seleção de genótipos desejáveis.

$\mathrm{Na}$ Tabela 4, encontram-se as correlações simples obtidas entre os caracteres avaliados. Das associações obtidas entre o peso médio do fruto (PMF) e os demais caracteres, duas foram positivas e significativas ao nível $1 \%$ de probabilidade, $o$ rendimento de frutos por cacho (RFC) e o peso de frutos por cacho (PFC) e apenas uma (PTC) foi correlacionada ao nível de $5 \%$ de probabilidade. Porém, todas elas exibiram baixas magnitudes com valores de 0,$33 ; 0,24$ e 0,22 , respectivamente. Por outro lado, o número de frutos por cacho (NFC) e o número

TABELA 1 - Esquema da análise de variância para a avaliação de caracteres do cacho de açaizeiro. Belém -PA, 2000.

\begin{tabular}{|c|c|c|}
\hline F a tor de varia çã o & Grau de liberdade & Q uadrado m éd io \\
\hline Ge nótipos & $g-1$ & $\sigma_{e}^{2}+n \cdot \sigma_{g}^{2}$ \\
\hline C a chos & $c-1$ & $Q M_{c}$ \\
\hline
\end{tabular}

g: genótipos; c: cachos

TABELA 2 - Quadrados médios para seis caracteres do cacho avaliados em 30 genótipos de açaizeiro. Belém -PA, 2000.

\begin{tabular}{|c|c|c|c|c|}
\hline C aracteres & $\mathbf{Q} \mathbf{M}_{\mathrm{g}}$ & $\mathbf{Q}_{\mathbf{e}}$ & C V (\%) & M é d ia \\
\hline P eso total (kg) & $2,5438 *$ & 1,4669 & 49,7 & 2,44 \\
\hline P e so de frutos (kg) & $1,9981^{*}$ & 1,2172 & 58,1 & 1,90 \\
\hline $\mathrm{N}$ ú m e ro de ráquilas ( U n id.) & $1,4244^{* *}$ & 0,5400 & 7,8 & 88,6 \\
\hline P eso m é dio do fruto (g) & $0,3848 * *$ & 0,0488 & 15,4 & 1,4 \\
\hline
\end{tabular}

**: significativo ao nível de $1 \%$ de probabilidade; *: significativo ao nível de $5 \%$ de probabilidade

TABELA 3 - Estimativas para a variância entre genótipos $\left(\mathrm{s}_{\mathrm{g}}^{2}\right)$, variância do erro $\left(\mathrm{s}_{\mathrm{e}}^{2}\right)$, coeficiente de repetibilidade (r), coeficiente de determinação $\left(\mathrm{R}^{2}\right)$ e número de medições necessárias $\left(\mathrm{h}_{\mathrm{o}}\right)$ obtidas para seis caracteres do cacho em 30 genótipos de açaizeiro da Coleção de germoplasma da Embrapa Amazônia Oriental. Belém -PA, 2000

\begin{tabular}{|c|c|c|c|c|c|}
\hline Caracteres & $\sigma_{g}^{2}$ & $\sigma^{2} \mathrm{e}$ & $\mathbf{r}$ & $\mathbf{R}^{2}$ & $\eta_{\circ}$ \\
\hline Peso total (P TC) & 0,2692 & 1,4669 & 0,1550 & 42,3 & 104 \\
\hline Peso de frutos (PFC) & 0,1952 & 1,2172 & 0,1382 & 39,1 & 118 \\
\hline Número de frutos (NFC) & 27,0143 & 98,4806 & 0,2152 & 52,3 & 69 \\
\hline Núm ero de ráquilas ( $\mathrm{N}$ C) & 0,2211 & 0,5400 & 0,2905 & 62,0 & 46 \\
\hline Peso m é dio do fruto (PMF) & 0,0840 & 0,0488 & 0,6328 & 87,3 & 11 \\
\hline Rendimento de frutos (RFC) & 0,0002 & 0,0064 & 0,0303 & 11,10 & 138 \\
\hline
\end{tabular}


TABELA 4 - Correlação simples entre seis caracteres do cacho avaliados em 30 genótipos de açaizeiro. Belém -PA, 2000

\begin{tabular}{|c|c|c|c|c|c|}
\hline C aracteres & P T C & P F C & N F C & N R C & P M F \\
\hline Peso de frutos (PFC) & $0,99^{* *}$ & & & & \\
\hline Número de frutos (NFC) & $0,91^{* *}$ & $0,92^{* *}$ & & & \\
\hline Núm e ro de ráquilas ( $N R C$ ) & $0,52^{* *}$ & $0,46^{* *}$ & $0,48^{* *}$ & & \\
\hline P eso m édio do fruto ( $P M F)$ & $0,22^{*}$ & $0,24^{* *}$ & $-0,10^{\mathrm{ns}}$ & $-0,05^{n s}$ & \\
\hline Rendimento de frutos (RFC) & $0,59^{* *}$ & $0,68^{* *}$ & $0,63^{* *}$ & $0,05^{\mathrm{ns}}$ & $0,33^{* *}$ \\
\hline
\end{tabular}

PTC: peso total do cacho; **: significativo ao nível de $1 \%$ de probabilidade; *: significativo ao nível de $5 \%$ de probabilidade; ${ }^{*}$ :não significativo; N: 120 .

de ráquilas por cacho $(\mathrm{NRC})$ não foram correlacionados a esta variável. Oliveira et al. (2000) encontraram resultados semelhantes para esses caracteres quando analisaram a relação entre caracteres de produção em açaizeiros implantados nas mesmas condições. Dessa forma, acredita-se que, praticando seleção para o caráter PMF nessa palmeira, podem-se estar selecionando indivíduos promissores para frutos.

\section{CONCLUSÕES}

1. Os genótipos de açaizeiro estudados apresentam variabilidade genética para o peso médio do fruto (PMF), enquanto os demais caracteres são altamente influenciados pelo ambiente.

2. Nas condições do estudo, o caráter peso médio do fruto possui consideráveis coeficientes de repetibilidade e de determinação. 3. O número de cachos necessários por genótipo para uma seleção mais eficiente para peso médio do fruto, em nível de $95 \%$ de probabilidade, deve ser de onze cachos. Para o peso do cacho (PTC), peso de frutos (PFC) e rendimento de frutos (RFC), o número de cachos desejáveis é muito elevado, tornando-se impraticável.

4. Utilizando-se do peso médio do fruto como parâmetro de seleção fenotípica simples, podem-se obter bons ganhos genéticos.

\section{REFERÊNCIAS BIBLIOGRÁFICAS}

COSTA, J.G da; LEDO, A da S; OLIVEIRA, M. N de. Estimativas de repetibilidade de características de frutos de cupuaçuzeiro no Estado do Acre. Revista Brasileira de Fruticultura, Cruz das
Almas, v.19, n.3, p.313-318, 1997.

CRUZ, C.D; REGAZZI, A.J. Modelos biométricos aplicados ao melhoramento genético. Viçosa; UFV, Impr. Univ., 1997.390p.

FALCONER, D.O. Introduction to quantitative genetics. London: Longman, 1975.365p.

LOPES, M.T.G; LOPES, R; BRUCKNER, C.H. Repetibilidade de características do fruto de aceroleira (Malpighia emarginata DC.). In: SIMPÓSIO BRASILEIRO DE MELHORAMENTO DE FRUTEIRAS, 2., 2000, Viçosa, MG. Anais..., p. 128.

OLIVEIRA, M do S.P de; LEMOS, M.A; SANTOS, E.O dos; SANTOS, V.F. dos. Variação fenotípica em acessos de açaizeiro (Euterpe oleracea Mart.) para caracteres relacionados à produção de frutos. Belém: Embrapa-CPATU, 1998.23p. (Boletim de Pesquisa, 209).

OLIVEIRA, M do S.P de; LEMOS, M.A; SANTOS, V.F. dos; SANTOS, E.O dos. Correlações fenotípicas entre caracteres vegetativos e de produção de frutos em açaizeiro. Revista Brasileira de Fruticultura, Jaboticabal-SP, v.22, n.1, p. 1- 5, 2000.

SIQUEIRA, E.R de. Coeficiente de repetibilidade da produção de frutos do coqueiro comum. Pesquisa Agropecuaria Brasileira, Brasília, v.17, n.4, p. 573-574, 1982.

VENCOVSKY, R. Princípios de genética quantitativa. Piracicaba: ESALQ, 1973.97p. 